\title{
ENSO Effect on East Asian Tropical Cyclone Landfall via Changes in Tracks and Genesis in a Statistical Model
}

\author{
EMMI YONEKURA \\ Department of Earth and Environmental Science, Columbia University, New York, New York \\ TIMOTHY M. HALL \\ NASA Goddard Institute for Space Studies, New York, New York
}

(Manuscript received 6 September 2012, in final form 25 September 2013)

\begin{abstract}
Improvements on a statistical tropical cyclone (TC) track model in the western North Pacific Ocean are described. The goal of the model is to study the effect of El Niño-Southern Oscillation (ENSO) on East Asian TC landfall. The model is based on the International Best-Track Archive for Climate Stewardship (IBTrACS) database of TC observations for 1945-2007 and employs local regression of TC formation rates and track increments on the Niño-3.4 index and seasonally varying climate parameters. The main improvements are the inclusion of ENSO dependence in the track propagation and accounting for seasonality in both genesis and tracks. A comparison of simulations of the 1945-2007 period with observations concludes that the model updates improve the skill of this model in simulating TCs. Changes in TC genesis and tracks are analyzed separately and cumulatively in simulations of stationary extreme ENSO states. ENSO effects on regional (100-km scale) landfall are attributed to changes in genesis and tracks. The effect of ENSO on genesis is predominantly a shift in genesis location from the southeast in El Niño years to the northwest in La Niña years, resulting in higher landfall rates for the East Asian coast during La Niña. The effect of ENSO on track propagation varies seasonally and spatially. In the peak activity season (July-October), there are significant changes in mean tracks with ENSO. Landfall-rate changes from genesis- and track-ENSO effects in the Philippines cancel out, while coastal segments of Vietnam, China, the Korean Peninsula, and Japan show enhanced La Niña-year increases.
\end{abstract}

\section{Introduction}

There are many densely populated coastal regions that are susceptible to high fatalities and costly damage every year because of the frequent landfall of tropical cyclones (TC) that occur in the western North Pacific Ocean (WNP). Studies have shown that El Niño-Southern Oscillation (ENSO) strongly influences TC activity in the WNP. To better understand the TC landfall risk in this region, the relationship with ENSO must be considered. The most important change of WNP TCs with ENSO appears to be the shift in genesis location to the southeast during strong El Niño events and to the northwest during strong La Niña events (Chan 1985, 2000; Dong 1988; Lander 1994;

Corresponding author address: Emmi Yonekura, Columbia University Department of Earth and Environmental Science, 2880 Broadway, New York, NY 10025.

E-mail: ey2111@columbia.edu
Saunders et al. 2000; Wang and Chan 2002; Chia and Ropelewski 2002; Camargo et al. 2007a). The shift is explained by the location of the monsoon trough in the WNP shifting eastward during El Niño events (Lander 1994) along with a reduction in vertical wind shear (Clark and Chu 2002), which both dictate favorable conditions for genesis. This shift results in changes in the tracks of the subsequent TCs: those that form in the southeast have longer lifetimes and tend to recurve northward as compared with those that form in the northwest that move straight westward onto land (Wang and Chan 2002). Elsner and Liu (2003) further studied the ENSO effect on tracks and implications for landfall, finding that strong El Niño years correspond with northward recurving TC tracks, which are likely to make landfall on Japan, the Korean Peninsula, and northern China. During La Niña years, TC tracks move in a straighter westward path, putting the Philippines, southern China, and Vietnam at higher risk. The TC-track cluster analysis performed by

DOI: 10.1175/JAMC-D-12-0240.1 
Camargo et al. (2007c) for the WNP demonstrates that the different genesis regions associated with El Niño (La Niña) years are also associated with longer (shorter) tracks. One important implication is that El Niño-year TC tracks in the WNP have more of an opportunity to reach higher intensities as they travel over the warm tropical ocean given a genesis location closer to the equator and farther eastward. El Niño-year TCs may also reach higher latitudes when they curve northward, before making landfall (Camargo and Sobel 2005; Chan and Liu 2004; Camargo et al. 2007c). The last two effects are important when making a landfall risk assessment in terms of landfall intensity and landfall location.

The ENSO-track relationship has been primarily explained by shifts in genesis location. One thing that has not been examined extensively is the direct effect of ENSO on tracks. For instance, independent of genesis location, will a TC tend to move in a different direction given different ENSO states? In this study, we investigate this effect as well as the combined genesis-and track-ENSO effects to attribute the changes in landfall to each component. We fill a key gap in documenting the TC-ENSO relationship and its landfall implications. As much work has been done on improving ENSO prediction (e.g., Cane 1991; Fedorov et al. 2003; Goddard et al. 2010), a fuller understanding of the ENSO-TC relationship would allow for improved long-range probabilistic predictions of TC landfall activity (Vitart 2006; Vitart et al. 2007; Camargo et al. 2010). Here we present developments of a statistical track model that can simulate the way that East Asian landfall changes with ENSO.

The advantage of using a statistical track model is the ability to translate what the TC activity-climate state relationships mean in terms of risk to humans. Observational studies have used track shape (Elsner and Liu 2003; Camargo et al. 2007b,c) or track density (Wang and Chan 2002; Wu et al. 2004; Zhang et al. 2012) changes as a proxy for risk of TC landfall because data become sparse when considering only the observed landfalls on small coastal regions. Data limitations increase when considering different climate states. Most other ENSO studies divide the observed data into subsets of less than 10 years based on a threshold of an ENSO index. An important new aspect of our work is the use of stochastic simulations from a statistical track model that employs regression instead of creating subsets. Since the model is stochastic, its simulations are not identical to observed tracks. Our synthetic tracks are each unique but as an ensemble have similar statistical characteristics to the observed tracks. Another advantage of statistical models (Darling 1991; Drayton 2000; Vickery et al. 2000; James and Mason 2005; Emanuel et al. 2006; Rumpf et al. 2007;
Hall and Jewson 2007; Graf et al. 2009; Yin et al. 2009; Yonekura and Hall 2011) is the ability to explicitly simulate landfalls. Our track-modeling method allows for better landfall risk assessment for different ENSO states at a resolution of $100-\mathrm{km}$ coastal segments instead of a country-scale assessment (e.g., Wu et al. 2004; Zhang et al. 2012). In effect, one could choose a specific segment or a city of interest instead of using a general risk level for a larger region or country.

In previous work, Yonekura and Hall (2011, hereafter YH11) use a statistical track model to simulate the full life cycle of TCs in the WNP including an ENSO-dependent genesis component. Here, we describe the addition of ENSO dependence into the track-propagation component to the YH11 model. In YH11, the details of statistical model construction, validation, and simulation procedures are presented. The genesis component depends on ENSO using local Poisson regression of the local genesis count time series on the July-October (JASO) Niño-3.4 index. The model was able to capture the geographic characteristics of landfall in its simulations of the historical period, as well as replicate the spatial distributions of the observed track trajectories and track densities.

One important aspect that was missing from the YH11 model is the seasonality of WNP TCs. Chan (2000) has shown that the effect of ENSO on WNP TC tracks varies by month. For instance, in October of a strong El Niño event, tracks will move more northward, avoiding Japan, because of midlevel westerlies around $40^{\circ} \mathrm{N}$. In the same month for a strong La Niña, it is shown that tracks move westward in a straight path at lower latitudes because of easterly midlevel wind anomalies. Chia and Ropelewski (2002) documented the seasonality in the mean genesis location in the WNP, which moves from the southeast in March $\left(5^{\circ} \mathrm{N}, 158^{\circ} \mathrm{E}\right)$ to the west in June $\left(135^{\circ} \mathrm{E}\right)$, to the northeast in the boreal summer $\left(17^{\circ} \mathrm{N}\right)$, and then back south-southeast in December $\left(8^{\circ} \mathrm{N}\right)$. The monsoon trough moves in the peak typhoon season as well as with ENSO events, which explains the genesis-location shift. In the Camargo et al. (2007b,c) WNP track cluster analysis, it is also shown that different clusters have different seasonal occurrence rates as well as different seasonal periods of positive or negative correlation with ENSO. In this study, we include the effects of seasonality in the genesis and track components by using seasonally varying predictors, another improvement from the YH11 model.

In the following section, we describe the methods used to incorporate ENSO and seasonal dependence into the model, run simulations, and discuss the model uncertainty. Then, in section 3, we evaluate the model, describe the effect of ENSO on TC tracks, and examine 
simulations to attribute track and genesis effects on landfall rates. Section 4 concludes with a summary and discussion of the results.

\section{Methods}

\section{a. Data}

As in YH11, the TC track data used to construct the statistical track model are from the International BestTrack Archive for Climate Stewardship (IBTrACS; Knapp et al. 2010) database of 6-hourly storm positions. The storms from 1945 to 2007 are used for consistency with YH11.

To define the state of ENSO for a given year, the JASO Niño-3.4 index (anomalies from the 1950-79 monthly climatology; Barnston et al. 1997) is used and will be hereafter referred to as the ENSO index. We acknowledge the recent studies of the central equatorial Pacific warming type of ENSO-an El Niño Modoki event (Ashok et al. 2007) — and would like to distinguish here that we are considering the conventional El Niño phenomenon defined by the first EOF from equatorial Pacific sea surface temperature (SST). The Niño-3.4 and Niño-3 indices are both well correlated with the principal component associated with the first EOF, and there is little difference in model results when using either index.

Seasonally varying predictors are used in both the genesis and track-propagation component of the model. The main purpose of adding SST as a parameter in the genesis component and midlevel winds in the track-propagation component is to include the effect of seasonality on WNP TCs, thus we use climatological predictors. The interaction term between the climatological predictor and ENSO index represents any existing correlation between the two that affects the TC model component, for example, if ENSO state affects seasonality or if the season affects the ENSO impact on TC activity. ENSO has known effects on the atmospheric circulation and SST in the WNP. At the midlevel troposphere, an El Niño event is associated with a cyclonic anomaly over Southeast Asia, which enhances the westerly jet along the coast (Wang et al. 2000). WNP SST, especially in $5^{\circ}-20^{\circ} \mathrm{N}, 130^{\circ}-155^{\circ} \mathrm{E}$, is negatively correlated with the Niño-3.4 index though its variability is considerably less than the index region (Chan and Liu 2004). We believe that our model captures these effects on TCs indirectly through the ENSO index predictor. We assume the physical environmental changes are implicit in the observed TC behavior.

For the genesis component, we use the monthly annualcycle climatology of SST from the National Oceanic and Atmospheric Administration (NOAA) extended reconstructed SST, version 3 (ERSST V3), data (Smith and Reynolds 2004) provided by the NOAA/Office of Oceanic and Atmospheric Research/Earth System Research Laboratory/Physical Sciences Division (OAR/ ESRL PSD) in Boulder, Colorado (the data can be found at http://www.esrl.noaa.gov/psd). In the track-propagation component of the model, the 500-hPa daily annualcycle climatology of zonal winds is used from the National Centers for Environmental Prediction-National Center for Atmospheric Research (NCEP-NCAR) reanalysis for the years from 1948 to 2007 (Kalnay et al. 1996).

The choice of 500-hPa zonal winds as a predictor for the track component is motivated by Chan and Gray (1982), who studied which wind levels most influence track displacement and speed. They found that the midlevel winds $(700,600$, and $500 \mathrm{hPa})$ have the most influence. Chan (2000) then used 500-hPa wind anomalies to explain changes in WNP TC tracks associated with different ENSO phases. Wu et al. (2004) made composites of the different ENSO-state 500-hPa winds to show that changes in landfall for El Niño and La Niña relate to the 500-hPa subtropical ridge. More recently, Zhang et al. (2012) looked at the 500-hPa wind-field anomalies and found mechanisms for ENSO-TC track effects. Looking at the NCEP-NCAR reanalysis data, the 500-hPa winds in the WNP follow a seasonal cycle. In December-February (DJF), there are strong westerlies in the midlatitudes poleward of $20^{\circ} \mathrm{N}$ and weaker easterlies in the tropics. In March-May (MAM), the band of westerlies moves northward, especially in the eastern part of the basin. Then, in June-August (JJA), the equatorial easterlies strengthen, and there is also more northward motion toward the East Asian coast. By October, the midlatitude westerlies begin to shift back southward to $20^{\circ} \mathrm{N}$. Here, we choose to use the 500-hPa winds as a first effort to incorporate a physicsbased parameter in track-propagation modeling.

\section{b. Genesis}

The genesis component of the model simulates the number of TCs per year and the day of year and formation location of each TC, all of which may be sensitive to ENSO. The total number is simulated by a random draw from a WNP-wide Poisson distribution whose rate is determined by Poisson regression of WNP-wide annual counts on the ENSO index (Sabbatelli and Mann 2007; YH11). The determination of the location of a genesis event is as in YH11: local Poisson regression is performed using annual formation count data inside data circles whose size is determined by out-of-sample likelihood maximization. The spatial field of Poisson rates is normalized to create a $1^{\circ} \times 1^{\circ}$ spatial probability 

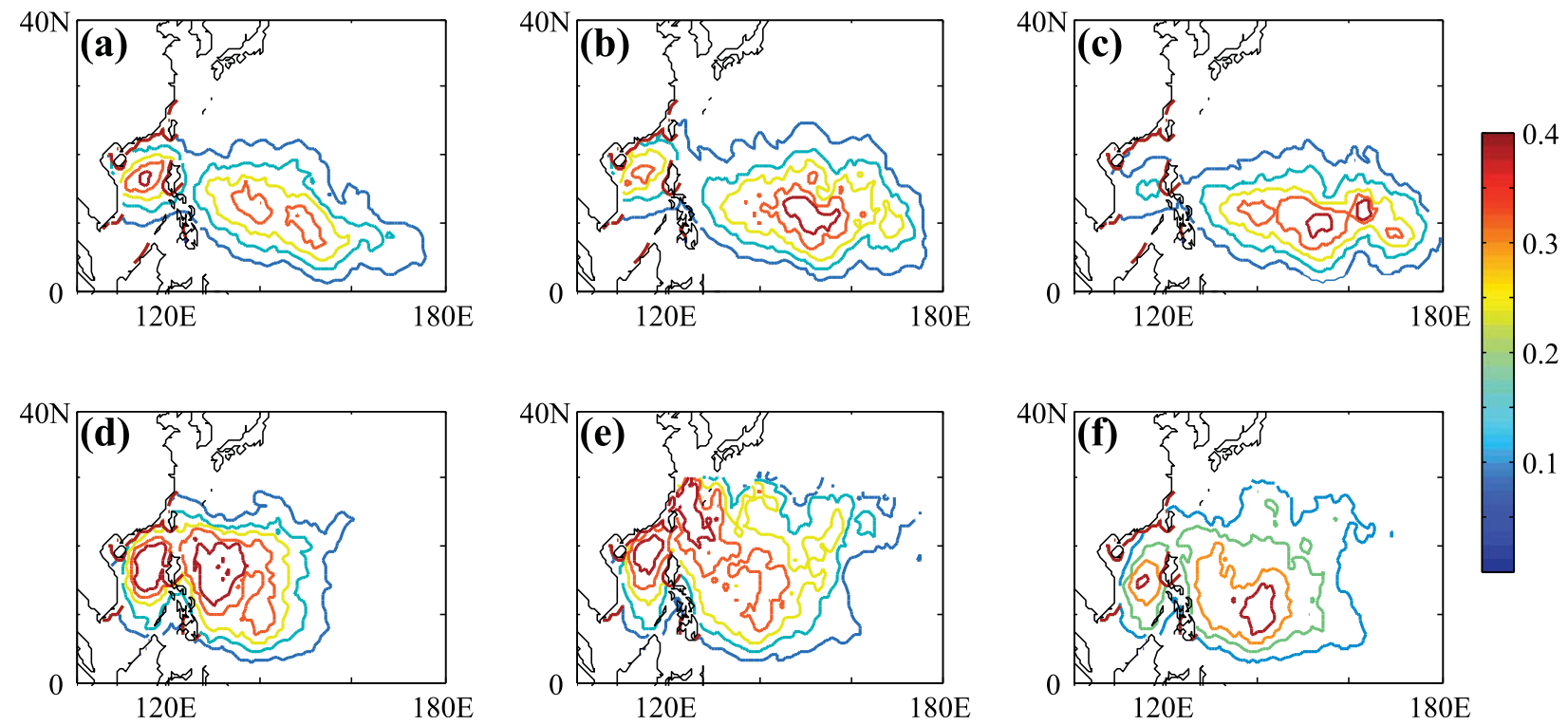

FIG. 1. Local Poisson rates calculated from the genesis model for El Niño (EN) and La Niña (LN) years (ENSO index values of +2 and -2 , respectively). Select months that specify a monthly climatological SST are shown: (a) June EN, (b) August EN, (c) October EN, (d) June LN, (e) August LN, and (f) October LN. Red contours outline regions with highest expected genesis rates.

density function (PDF), and a random draw from the PDF determines the location for each simulated TC. Further details can be found in YH11.

We have added seasonality to the genesis model of YH11. We include the local monthly annual-cycle climatology of SST as an independent predictor variable in the regression. We randomly select a day of year, and the SST value of that day fixes a Poisson rate for a formation event. Then we sample the probability and repeat until an event is realized. An ENSO-SST interaction term is also used as a predictor to account for the effect of yearly ENSO state on seasonality. Equation (1) shows how to calculate a local Poisson rate $\lambda$ :

$$
\begin{aligned}
\lambda(r, a, m)= & \exp \left[\beta_{0}(r)+\beta_{\mathrm{ENSO}}(r) \times \operatorname{ENSO}(a)\right. \\
& +\beta_{\mathrm{SST}}(r) \times \operatorname{SST}(r, m)+\beta_{\mathrm{ENSO} \times \mathrm{SST}}(r) \\
& \times \operatorname{ENSO}(a) \times \operatorname{SST}(r, m)],
\end{aligned}
$$

where $r$ stands for a grid location, $a$ specifies a year, $m$ specifies a month, each $\beta$ is a local regression coefficient at $r, \operatorname{ENSO}(a)$ is the ENSO index for year $a$, and $\operatorname{SST}(r, m)$ is the SST at location $r$ and month $m$. As in YH11, the shift in dominant genesis region to the southeastern part of the WNP for El Niño years and to the northwest for La Niña years is observed for different inputs of $\operatorname{ENSO}(a)$, shown in Fig. 1. The shift is most prominent between June and October, when $76 \%$ of WNP TCs occur. In Fig. 1, the genesis Poisson rate fields for June, August, and October in different ENSO states are shown. The subtle seasonal shifts of high-Poisson-rate regions in Fig. 1 agree with the seasonal cycle of mean genesis location from Chia and Ropelewski (2002). Our model now captures both ENSO and seasonal effects on WNP TC genesis.

\section{c. Tracks}

The track component of the model simulates the 6-hourly increment of a TC position as a function of location, day of year, and ENSO state. The basic methodology is identical to that described in YH11, but here we have added seasonality and ENSO dependence. Our incorporation of ENSO and seasonality is guided by the work of Chan and Gray (1982) and Chan (2000), who analyzed ENSO-track relationships month by month and attributed the ENSO effect to anomalies in the 500-hPa winds. They demonstrated that the effect of ENSO on TCs in the WNP evolves through the season. Thus, the ENSO index and an annual cycle of 500-hPa winds are used as parameters to incorporate seasonality in track propagation. The wind data come from daily climatological zonal winds interpolated to a $1^{\circ}$ grid. We found the meridional winds to be insignificant as a predictor based on a two-tailed Student's $t$ test. The meridional winds are not used to avoid modeling a relationship with a high degree of uncertainty, which could introduce a model bias. The lack of significance may be due to the meridional displacement being governed more by TC size and latitude. 

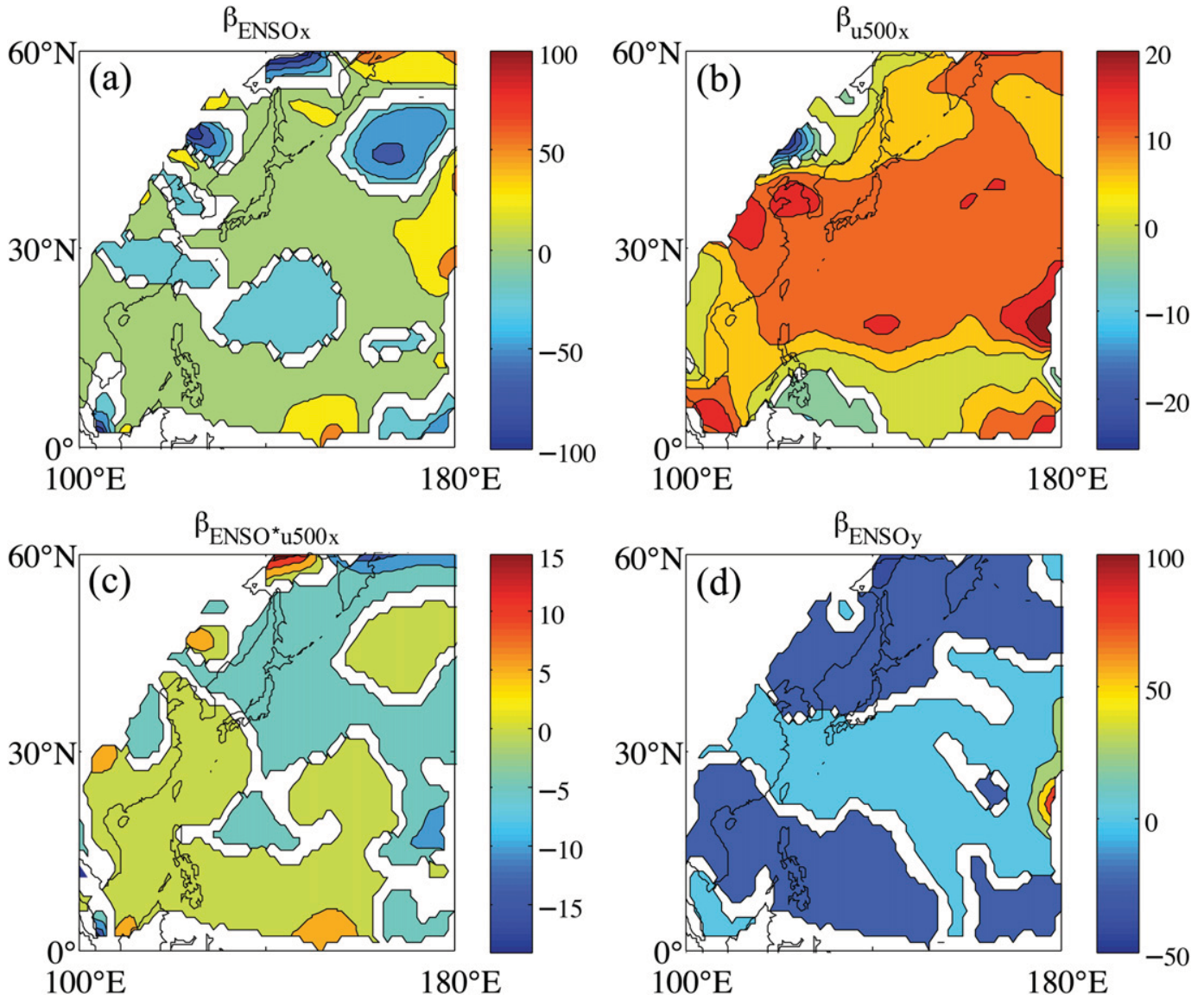

FIG. 2. Local regression coefficients used in the track-propagation model component in the zonal $(x)$ and meridional $(y)$ directions. White areas indicate where the regression coefficient is not significant. (a) $\beta_{\mathrm{ENSO} x}$ and (d) $\beta_{\text {ENSO } y}$ are the coefficients that relate the mean track to the current-year ENSO index. (b) $\beta_{u 500 x}$ relates the zonal mean track component to the daily climatological 500-hPa zonal winds. (c) $\beta_{\mathrm{ENSO} \times u 500 x}$ accounts for the correlated components of the ENSO index and 500-hPa zonal winds that effect the zonal mean track displacement.

The IBTrACS 6-hourly zonal and meridional displacement values are linearly regressed locally on their respective predictors. The distance weight determining the locality of regression is a Gaussian kernel with a length scale of $300 \mathrm{~km}$. The length scale is determined objectively by out-of-sample likelihood maximization. At each $1^{\circ}$ grid point, we archive values of the regression coefficients from the equations:

$$
\begin{aligned}
\overline{d X}(r, a, d)= & \beta_{0 x}(r)+\beta_{\mathrm{ENSO}}(r) \times \operatorname{ENSO}(a) \\
& +\beta_{u 500 x}(r) \times u_{500}(r, d)+\beta_{\mathrm{ENSO} \times u 500 x}(r) \\
& \times \operatorname{ENSO}(a) \times u_{500}(r, d) \text { and }
\end{aligned}
$$$$
\overline{d Y}(r, a)=\beta_{0 y}(r)+\beta_{\mathrm{ENSO} y}(r) \times \operatorname{ENSO}(a),
$$

where $\overline{d X}(r, a, d)$ and $\overline{d Y}(r, a)$ are the mean displacements in the zonal and meridional directions, respectively, at grid point $r$, year $a$, and day $d$. $\operatorname{ENSO}(a)$ is the ENSO index for the year specified by year $a$, and $u_{500}(r, d)$ is the 500-hPa zonal wind at grid point $r$ and day $d$ from a daily climatology. Note that ENSO is only included as an effect for the mean track increment. In full simulations TCs propagate according to Eqs. (2) and (3) in the mean, and the residuals about the mean are modeled stochastically, as described in YH11: a bivariate anisotropic correlated normal, with lag-1 autocorrelation for the standardized errors.

The resulting fields of coefficients from regression Eqs. (2) and (3) are shown in Fig. 2, in which the contours are color filled where the coefficients are significantly different than zero at the $95 \%$ confidence level according to a two-tailed Student's $t$ test at every grid point. ENSO significantly affects TC mean tracks. In Fig. 2a, the $\beta_{\text {ENSO } x}$ regions of negative values near $20^{\circ} \mathrm{N}$, $150^{\circ} \mathrm{E}$ and $45^{\circ} \mathrm{N}, 150^{\circ} \mathrm{E}$ indicate that positive (negative) ENSO tends to enhance (inhibit) the westward motion 
of TCs in an important region for landfall consideration. In this region, as well as in the rest of the basin, positive (negative) ENSO tends to inhibit (enhance) the northward motion of TCs (Fig. 2d). Figure 2b shows $\beta_{u 500 x}$, which not surprisingly shows a positive relationship between the midtropospheric zonal steering wind and TC motion. Finally, $\beta_{\text {ENSO } \times u 500 x}$ (Fig. $2 c$ ) reveals some coupling between ENSO and $u_{500}$, particularly at midlatitudes. For positive (negative) ENSO the TC motion enhances (inhibits) the relationship between $\mathrm{TC}$ motion and the seasonal steering winds.

\section{d. Lysis}

Lysis, or death, of each TC is determined by comparing a random uniform draw to a predetermined PDF of historical lysis probability at every track step. Land and ocean lysis is strictly separated according to a $0.25^{\circ}$ land-ocean mask. See YH11 for details.

\section{e. Simulations}

Our goal is to estimate the effects of ENSO on East Asian TC landfall via genesis and track propagation. Separation of the two ENSO-landfall effects is investigated by running 10000 -yr simulations at stationary values of the ENSO index using different model versions. Here, we present the simulations using the ENSO index values of +2 and -2 anomaly values from the $1950-79$ base period. The $10000-y r$ duration is required to converge on mean landfall rates at high geographic resolution. The four model versions are 1) no dependence on ENSO; 2) genesis dependence on ENSO ("GenesisOnly"); 3) track dependence on ENSO ("Track-Only"); and 4) genesis and track dependence on ENSO ("Both").

We also evaluate each of the four model versions against historical landfalls. For this purpose, a separate set of simulations is performed: 500 realizations of the historical period 1945-2007 for each model version using the historical time series of the ENSO index. The mean and spread of each ensemble is compared to the historical landfall rates. The model is unbiased to the extent that the historical landfall rates appear as a typical member of the 500-member synthetic set.

\section{f. Uncertainty and significance}

There are two types of uncertainty to document. One is the uncertainty due to the stochastic nature of our simulations. For example, a single 1-yr simulation in a +2 ENSO state will have landfalls along the coast that differ from those of another 1-yr +2 ENSO simulation, mimicking the chaotic nature of TCs. When we perform a multiyear simulation, we compute the mean value of a diagnostic (e.g., landfall rate) and the spread about the mean. How well do we know the mean? We perform long simulations for a fixed ENSO state to reduce the uncertainty of the mean due to finite simulation length. We find that $10000-y r$ simulations are required to converge on mean model landfall rates at high resolution along the coast. We cannot, however, reduce the uncertainty due to the finite historical data on which the model is constructed. This is the second type of uncertainty, and it is crucial to document this uncertainty to say whether the relationships we uncover between ENSO and a mean diagnostic value are significant.

To determine the second type of uncertainty, we perform a generalized jackknife significance test (Tukey 1958). We reconstruct the genesis and track models 100 times, each time using a random $\sim 80 \%$ of the IBTrACS years (50 years out of 63). For each of the 100 jackknife members, we run 10000-yr simulations at extreme ENSO states and compute the mean of the diagnostic (e.g., landfall rates along the coast). We then take the difference between +2 and -2 ENSO mean values for each jackknife member. If at least $97.5 \%$ of the difference values are all positive or all negative, then we call the ENSO effect significant for that diagnostic.

\section{Results}

In this section we first evaluate the model by comparing historical simulations to direct observations. We then examine the impact of ENSO on WNP TC tracks and landfall, first by illustrating the ENSO effect on mean tracks, and then by performing full stochastic TC simulations at stationary strong ENSO states and examining the landfall rates. Landfall rates from three different model versions are used to isolate the effects of ENSO on landfall via genesis and tracks.

\section{a. Model evaluation and comparison}

We now evaluate the model by comparing simulation results to observations using two diagnostics: 1) life spans at extreme ENSO states and 2) landfall rates over the historical period. We calculate TC life spans from IBTrACS and find an average of 9.3 days for years when the ENSO index is greater than 1 (El Niño) and 6.8 days when the ENSO index is less than -1 (La Niña). Using the "Both" model version (ENSO-dependent tracks and genesis), we obtain a mean life span of 8.6 days during El Niño and 6.8 days during La Niña, in close agreement with the direct observations. Repeating the calculation with Genesis-Only and Track-Only model versions indicates that the ENSO influence on genesis causes the change in life span. Genesis-Only has a mean life span of 8.3 (6.3) days for El Niño (La Niña). 


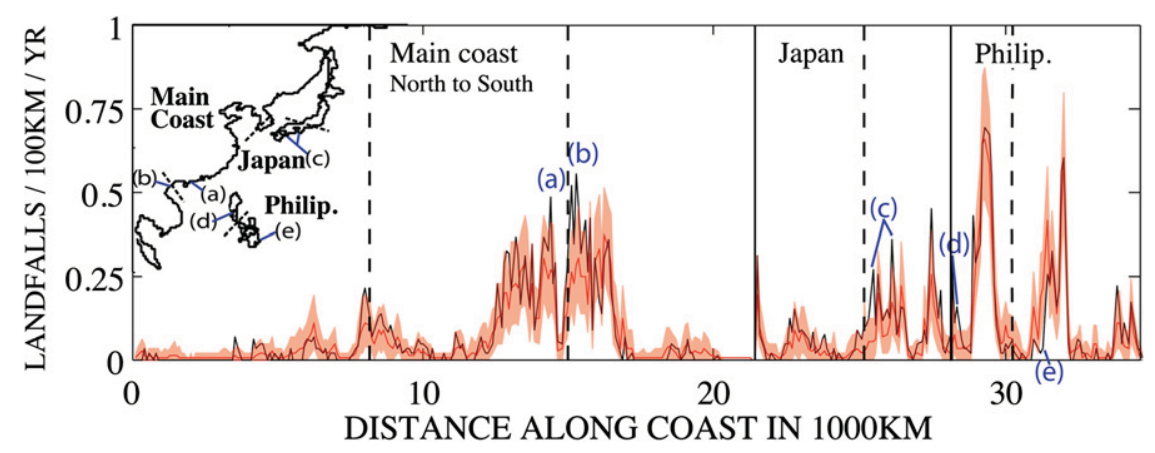

FIG. 3. The ensemble-mean landfall rates for each 100-km segment from simulations of the historical period (1945-2007) are shown with the solid red line. The red shading indicates the inner $95 \%$ spread of landfall rates over the 500 -member ensemble. The Both model configuration was used, having ENSO-dependent genesis and tracks. Landfall rates from IBTrACS are in black. Letters highlight where the IBTrACS landfall rates fall outside the model inner $95 \%$ spread, corresponding to the letters in the inset. The map inset shows the coast defined by 100-km segments used in the landfall diagnostic, separated into larger regions: main coast, Japan, and the Philippines. The dashed lines correspond to the dashed lines in the main panel.

Track-Only has a mean life span of 7.5 (7.7) days. La Niña TCs originate, on average, closer to the coast and, thus, have a shorter life span before making landfall. We note that others (Wang and Chan 2002; Camargo and Sobel 2005) find the same sign in ENSO life-span change, though they use different ENSO thresholds. Camargo et al. (2007c) calculate the median lifetime for track types, or clusters, that occur more during El Niño states. Their result is higher than our average El Niño lifetime by about 2-4 days. The median lifetime for their cluster occurring most with La Niña has about the same value as our La Niña average.

To evaluate the landfall characteristics of the model, we perform 500 full stochastic simulations of the historical period 1945-2007. We define a landfall as when a track crosses a $100-\mathrm{km}$ coastal segment from ocean to land for the East Asian regions shown in the Fig. 3 inset. Figure 3 shows the average annual landfall rates per $100 \mathrm{~km}$ over the 500 -member ensemble, the inner $95 \%$ spread about the mean, and the rates directly computed from IBTrACS. Landfall rates vary enormously by region, depending on the orientation of the coast with respect to mean tracks and the proximity of the coast to regions of high activity. Peak landfall rates occur in Southeast Asia, southern China, and the Philippines.

The model is in close agreement with observed landfall rates. In most regions the historical landfall rate falls inside the $95 \%$ spread about the mean, indicating no model bias. There are, however, a few scattered regions of bias that are identified with letters that correspond to the coastal segments highlighted on the Fig. 3 map inset. Just north (Fig. 3a) and south (Fig. 3b) of the Vietnam-China border, there are local landfall peaks where the model is biased low. Small regions of low bias also occur on the southeastern coast of Japan (Fig. 3c) and the western coast of the northern Philippines (Fig. 3d). The eastern coast of the southern Philippines (Fig. 3e) displays a small region of high bias. Despite these small biases, overall the analysis confirms that the model is able to capture the geographically complex behavior of TC landfall in the WNP. Furthermore, the inclusion of ENSO reduces model bias compared to a model version with no ENSO sensitivity (not shown). One possible reason for the bias is that the lysis model does not depend on the intensity of the storm. When an intensity component is added to the model, lysis will be determined more realistically by considering intensity. Another possible explanation is that the landfall rates from 500 simulations of the 63-yr historical period may not be sufficient for the model to simulate the full range of possible landfalls. However, it could also be that the "biases" are spurious. In any 63-yr realization of the stochastic process, one expects several local landfallrate excursions beyond the $95 \%$ band about the mean, if enough regions are queried.

\section{b. ENSO effect on mean tracks}

The modulation of TC tracks in the WNP due to ENSO is examined by looking at mean tracks for ENSO index values of +2 and -2 . Mean tracks are the trajectory followed as a result of the local regression of Eqs. (2) and (3), without any stochastic modeling of residuals. This is, in effect, the average trajectory that a TC would follow as it moves through the basin starting from a specified location and date. We compare mean tracks that originate from the same point to exclude the indirect influence of ENSO on tracks via shifts in genesis site. 
August 15
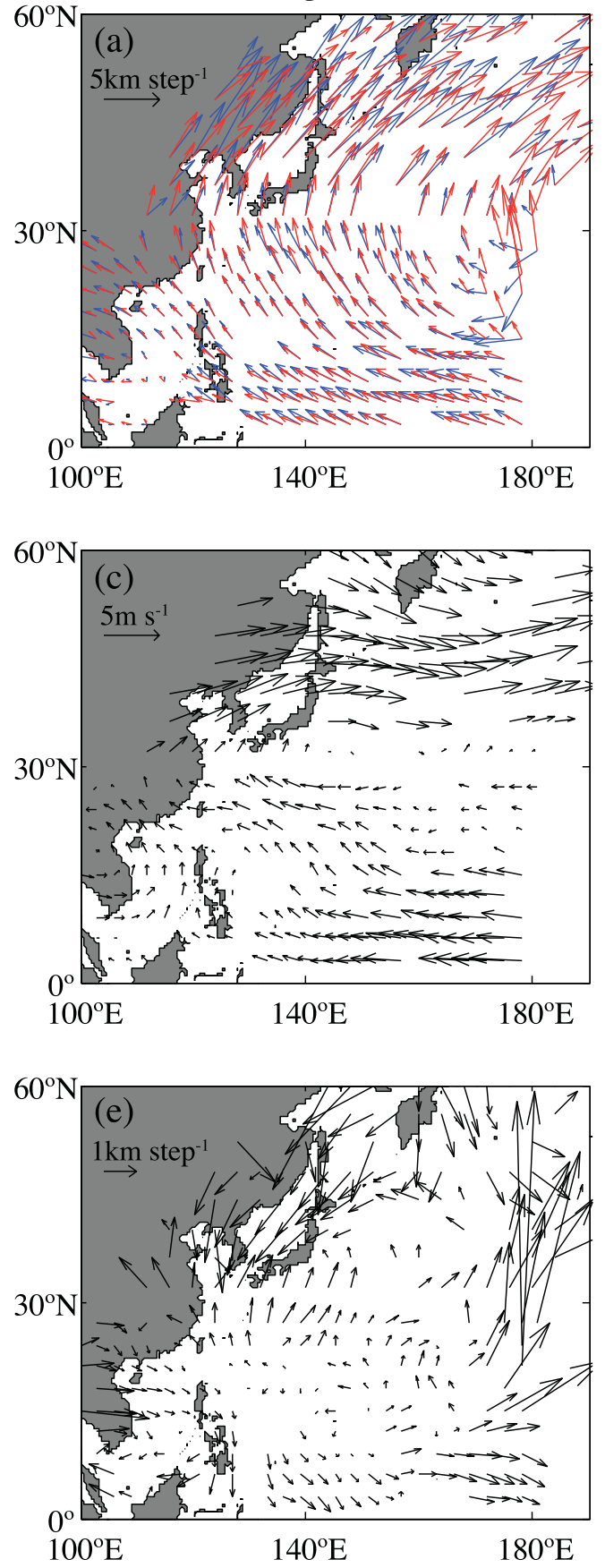

\section{October 15}
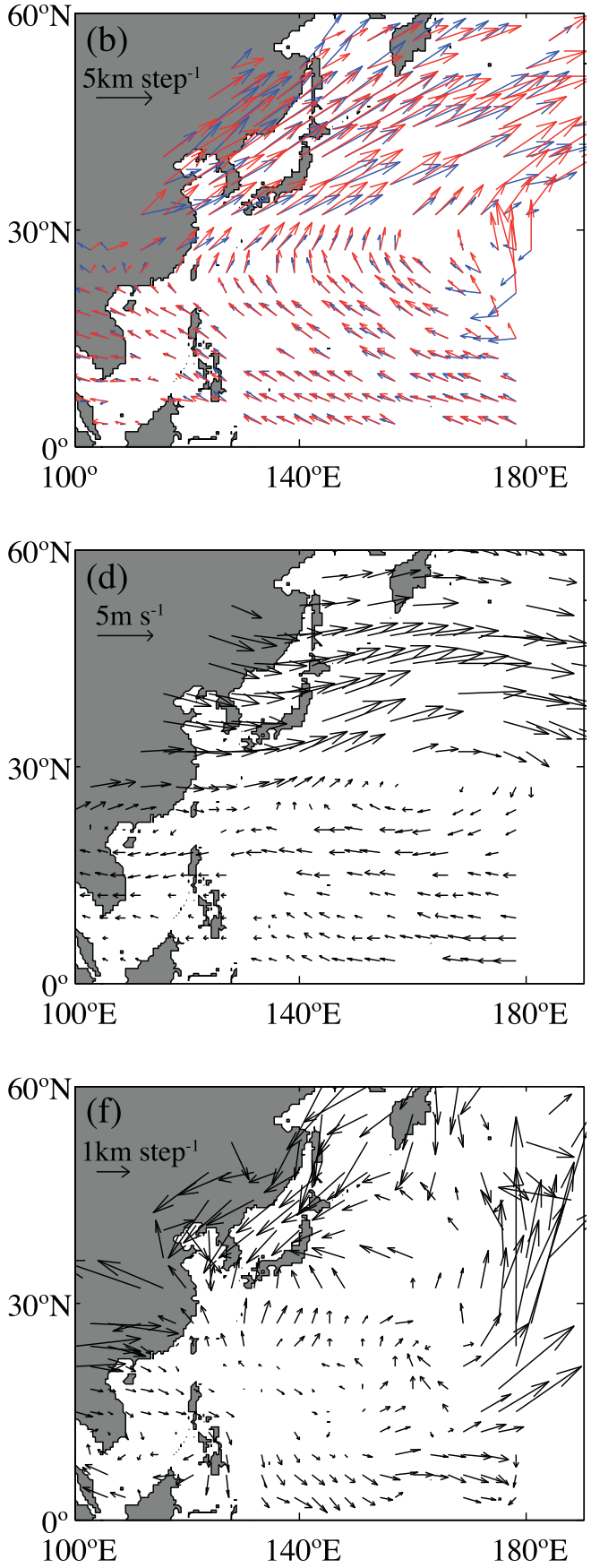

FIG. 4. Local mean-track displacement vectors for ENSO-index values of +2 (red) and -2 (blue) shown for (a) 15 Aug and (b) 15 Oct, which each have distinct climatological daily 500-hPa zonal winds. Vectors are only shown where the local regression coefficients are significant. (c),(d) 500-hPa wind vectors from the daily climatology for each respective day. (e),(f) The difference between red and blue vectors from (a),(b), respectively, which highlights the regions where the local mean displacement changes with ENSO state. The units for the vectors in (a),(b),(e), and (f) are kilometers per step, where "step" refers to the 6-h steps of the model. 
Aug 15 genesis
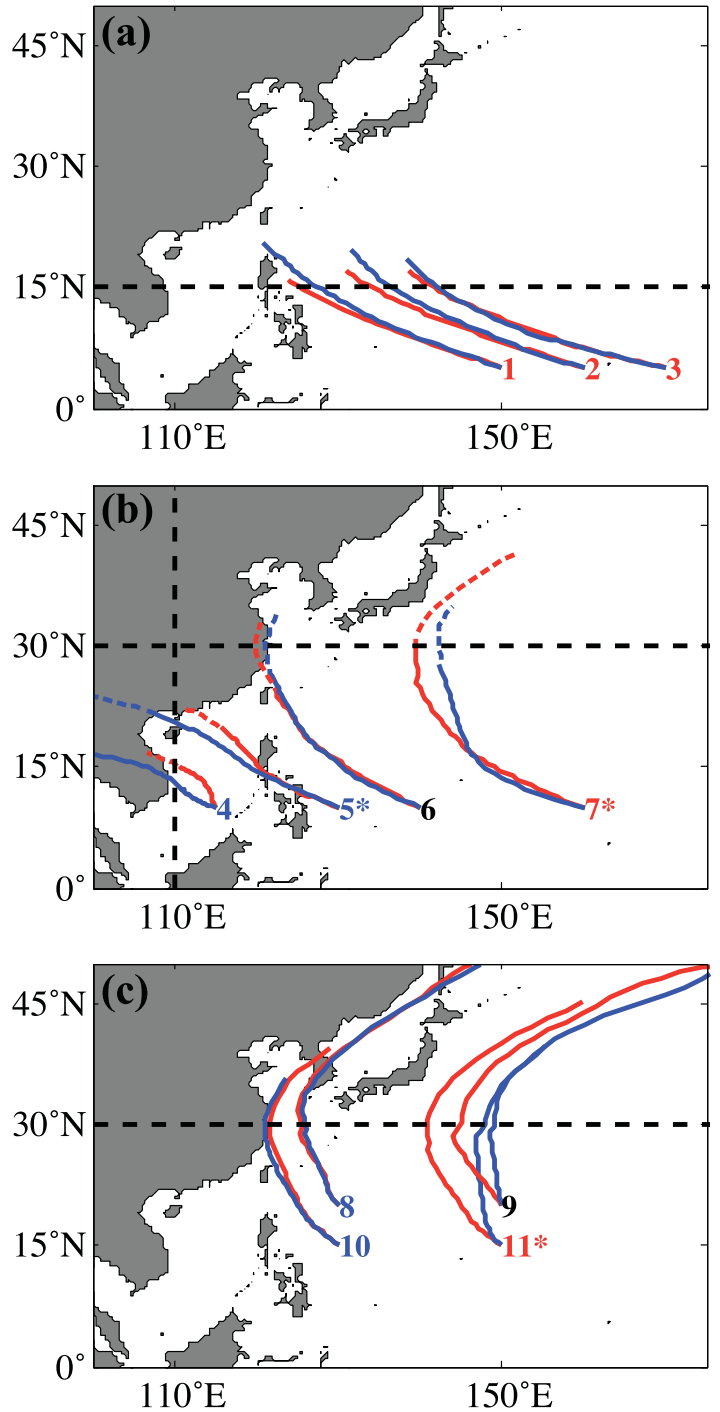

Oct 15 genesis
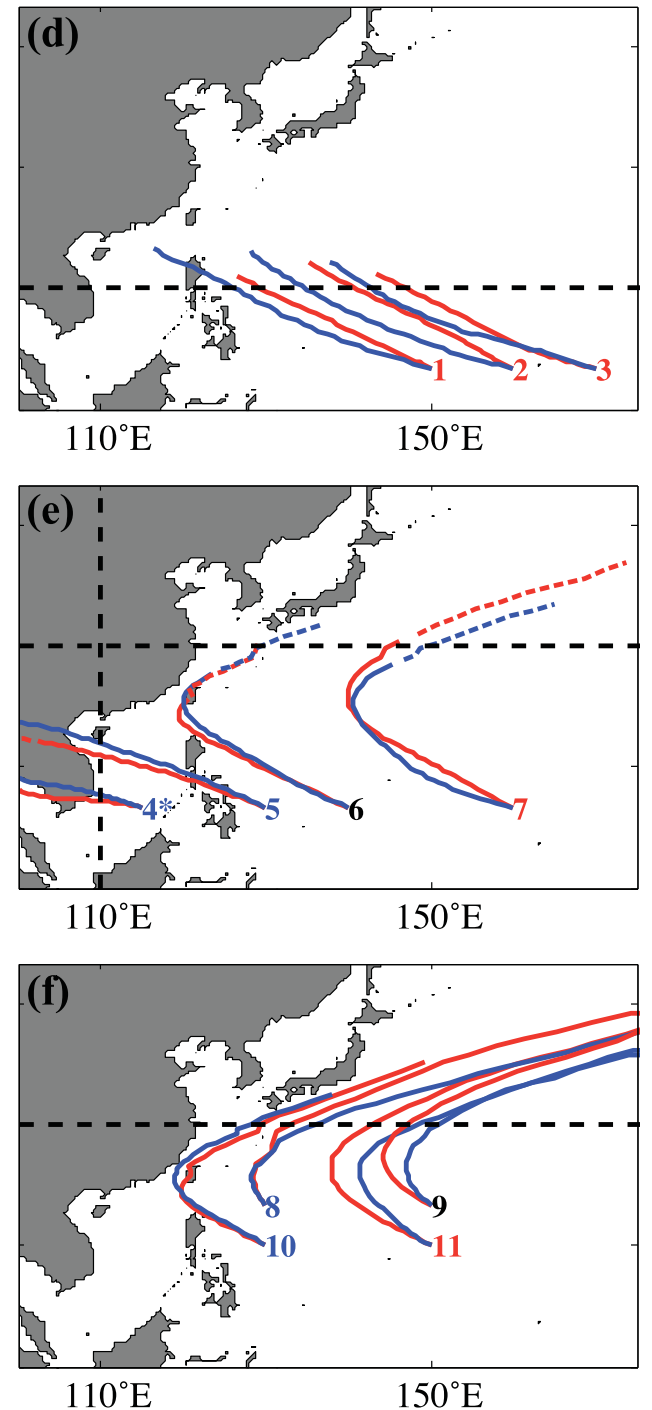

$110^{\circ} \mathrm{E}$

$150^{\circ} \mathrm{E}$

FIG. 5. Mean tracks initiated on (a)-(c) 15 Aug and (d)-(f) 15 Oct from numbered genesis locations with ENSO indices of +2 (red lines) and -2 (blue lines) for extreme El Niño and La Niña years, respectively. The dashed portions of the red and blue lines indicate where the 10-day mean tracks were extended to reach the diagnostic line. The dashed black lines are used for a diagnostic that measures the difference in crossing location between ENSO states. The asterisks by genesis point numbers indicate that the resulting mean tracks have a significant shift, and the red (blue) number colors indicate that the genesis site is more likely to occur during an El Niño (La Niña) year.

Figures $4 \mathrm{a}$ and $4 \mathrm{~b}$ show the local mean displacement vectors for opposite ENSO states at two dates. The vector differences are highlighted in Figs. $4 \mathrm{e}$ and $4 \mathrm{f}$ and can be interpreted as showing the tendency of mean tracks in El Niño years relative to La Niña years. There are several key differences between mean-track vectors for the two ENSO states that are relevant to TC landfall risk, which are present in both months. At low latitudes (below $15^{\circ} \mathrm{N}$ ), El Niño (red) vectors have weaker westward components than La Niña (blue) vectors, resulting in a slower shuttling of TCs toward the Philippines and Vietnam. South of Japan, red vectors exhibit a stronger northward component. West of Japan and around the Korean Peninsula, blue vectors have stronger northeastward magnitudes. Examining the 500-hPa wind from the daily climatology for the two dates in Figs. $4 c$ and $4 d$ shows the expected steering winds with easterlies in the tropics and stronger westerlies in the midlatitudes. Differences between August and October winds here include a stronger northward component around the 
TABLE 1. Mean-track significance testing results for each genesis point and starting date. An "M" ("F") signifies that the mean track shift from opposite ENSO states was significant for a starting date on the fifteenth (first) day of the given month. Asterisks indicate months when the ENSO effect on mean tracks is of the opposite sign from the other months.

\begin{tabular}{llllllllllll}
\hline \hline & \multicolumn{10}{c}{ Genesis point } \\
\cline { 2 - 10 } & 1 & 2 & 3 & 4 & 5 & 6 & 7 & 8 & 9 & 10 & 11 \\
\hline July & $\mathrm{M}, \mathrm{F}$ & $\mathrm{F}$ & & & & & & & & $\mathrm{M}$ \\
August & $\mathrm{F}$ & $\mathrm{F}$ & & & $\mathrm{M}$ & $\mathrm{M}, \mathrm{F}$ & & & $\mathrm{M}$ \\
September & $\mathrm{F}$ & $*$ & $*$ & & & $\mathrm{~F}$ & & & \\
October & $*$ & $*$ & $*$ & $\mathrm{M} *$ & $*$ & & $\mathrm{~F}$ & & & \\
\hline
\end{tabular}

Philippines in August, stronger easterlies in the tropics in August, and strong westerlies reaching lower latitudes in October; all differences are reflected in the mean track behavior in Figs. 4a and 4b.

To better visualize the cumulative effect of these space- and time-dependent ENSO dependencies, we launch mean tracks of 10-day durations in +2 and -2 ENSO states from a set of fixed locations in July, August, September, and October. Figure 5 shows the tracks for August and October, while Table 1 additionally documents results for July and September. As a diagnostic of the ENSO effect we use the location where tracks cross lines of constant latitude and longitude. The ENSO difference in the location (degrees of latitude or longitude) is deemed to be significant or not according to our jackknife uncertainty tests described above. An asterisk by the genesis point in each figure indicates that the ENSO effect is significant at the $90 \%$ confidence level. Figure 6 provides examples of the jackknife test for the case of an insignificant ENSO effect and a significant ENSO effect.

These 10-day tracks can be divided into two broad categories, those that recurve, ultimately heading northeastward, and those that remain on a comparably straight northwestward trajectory. We find that both track categories occur in both ENSO states, and that there is little variation from one category to another through the season from a given genesis site. Genesis points $1-5$ had straight-moving tracks through the whole season, while genesis points 6-11 had recurving tracks through much or all of the season. The only variations occurred for genesis points 6 and 10, which had straight-moving tracks in July and recurving tracks in August-October.

ENSO influences the trajectory of both the straightmoving and recurving tracks. We summarize this influence by calculating the shift in location that mean tracks cross specified lines of longitude or latitude (dashed black lines in Fig. 5). The sign of mean track shifts with ENSO changes through the season for the straight movers but stays constant for recurving tracks
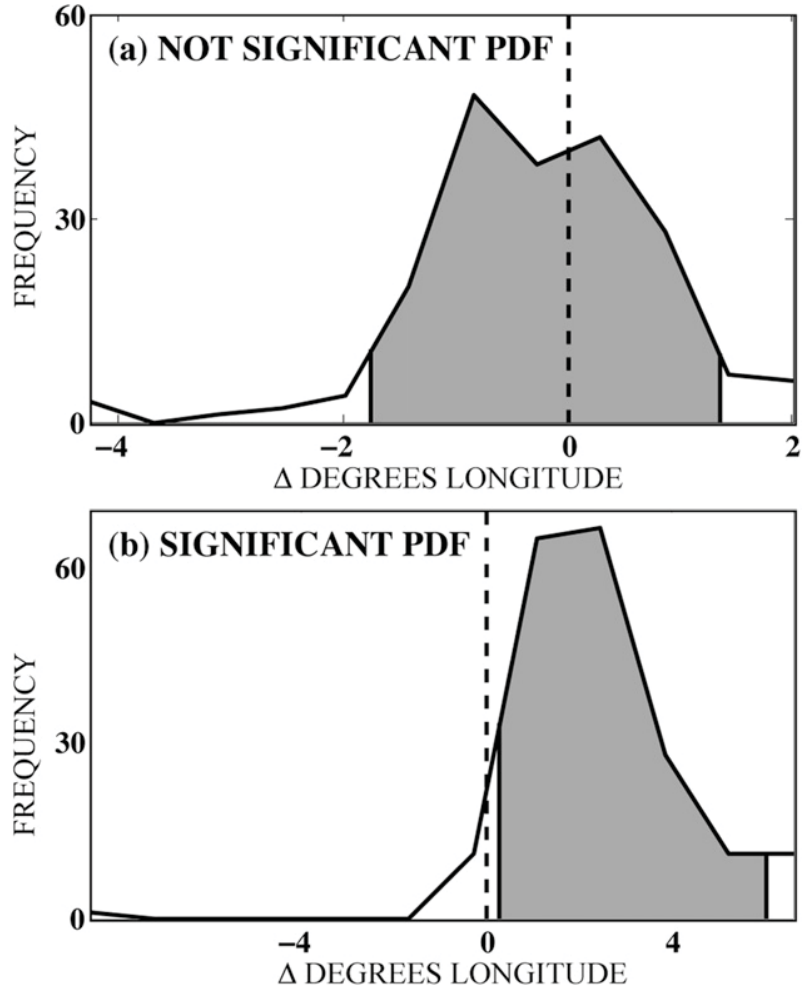

FIG. 6. Illustration of the significance test for the mean track shift between two ENSO states across diagnostic lines from Fig. 5. (a) The distribution of mean track shifts from a genesis site that results in an insignificant track shift: the inner $90 \%$ spread (shaded) bounds zero. (b) A similar distribution from another genesis site that produces a significant track shift.

(Table 1, asterisks). The ENSO shift for straight-moving tracks from genesis points 1-5 (Fig. 5) reverses from August to October: in August, El Niño (La Niña) mean tracks move more westward (eastward) and northward (southward), and the effect reverses in October. Most of the shifts of either sign are not significant, though for select genesis dates there is a significant ENSO shift for points 1, 2, 4, and 5 (Table 1). Most of the recurving mean tracks from genesis points 6-11 propagate farther westward during El Niño before recurving. However, only tracks from points 7 and 11 have significant shifts.

The straight-moving tracks pose the biggest landfall threat for the Philippines, Vietnam, and southern China. The shifts with ENSO result in changes in landfall location within these regions. However, the ENSO effect reverses throughout the season resulting in some cancellation of the net annual ENSO-track influence for these tracks. Recurving tracks threaten the coasts of Japan, the Korean Peninsula, and northern China. The El Niño westward shift of recurvature point enhances the chance of a landfall. 
Before running full stochastic simulations, we first consider the combined landfall effect of ENSO on genesis and tracks by using the mean tracks and the known shift in genesis region. On Fig. 5, genesis points $1,2,3,7$, and 11 have a higher likelihood of genesis during an El Niño year (red numbers), while genesis points 4, 5, 8, and 10 have a higher likelihood during a La Niña year (blue numbers) determined from Fig. 11 in YH11. Looking at combined effects by following the red (blue) tracks for likely El Niño (La Niña) genesis points, results are similar to those of Elsner and Liu (2003): landfall rates increase in the northern Philippines and Japan during an El Niño and increase on Vietnam, China, the Korean Peninsula, and the Philippines during a La Niña.

\section{c. ENSO and landfall rates}

We now turn to landfall rates using the fully stochastic model. The results for a neutral state (i.e., ENSO index of 0 ) are not shown, but the neutral landfall rates fall in between the two ENSO states shown for most of the East Asian coast. As shown by YH11, the Genesis-Only model produces higher landfall rates everywhere along the coast during La Niña (Fig. 7a). The genesisENSO effect on landfall rates is most pronounced on the main coast (defined in the Fig. 7c inset) near the China-Vietnam border, where there are higher landfall rates during La Niña (blue) than during El Niño (red). The result is expected: the region of primary genesis is shifted to the northwestern WNP during La Niña, closer to the coast. The genesis-ENSO effect is less pronounced for regions of low landfall rates, such as Indonesia-Malaysia and Kamchatka (not shown).

The Track-Only model shows more spatially varied ENSO effects at landfall (Fig. 7b). The ENSO-track effect is similar to the ENSO-genesis effect for southern China, Vietnam, and the northern Philippines, having higher rates during La Niña. However, Japan, the southern Philippines, and Thailand have higher rates during El Niño, with the most pronounced increase in the Philippines. There is no longer a difference in the Korean Peninsula and northern China. Furthermore, landfall rates overall are higher using Track-Only than Genesis-Only.

The landfall rates resulting from the inclusion of both track- and genesis-ENSO effects (the Both model) closely resemble those of the Genesis-Only model. Figure 7c shows that the ENSO effect on China, Vietnam, and the northern Philippines is enhanced because of the genesis and track effects combining. There is a less pronounced effect on Japan and little effect on the lowlandfall regions. For the southern Philippines, there is virtually no difference between ENSO states, showing that the track effect offsets the genesis effect for this area. The red and blue shading show the $95 \%$ confidence, or uncertainty, on the mean landfall rates from the generalized jackknife significance test. In many regions, the jackknife uncertainty overlaps for the two ENSO states.

To further highlight the significance of the ENSO effect for the Both model, the difference in landfall rates from El Niño to La Niña is plotted in Fig. 7d, including the jackknife uncertainty of this difference. The regions where the uncertainty excludes zero have a significant ENSO effect at the $95 \%$ confidence level. The western and southern coasts of the Korean Peninsula, parts of China, part of Vietnam, southern Japan, and small points on the northern tips of Japan and the Philippines meet this condition (highlighted in blue on the Fig. 7c map inset). In these regions we can say with $95 \%$ confidence that there are more landfalls on average during La Niña than during El Niño. These results differ slightly from Saunders et al. (2000), who use a different type of statistical model to determine the ENSO effect on East Asian landfall. They find that the only significant difference is a La Niña increase in landfall in the Philippines (around Manila) and Vietnam. The former contrasts with our result, though it is in an area where our model exhibits a low bias. Saunders et al. (2000) also find an increase in landfall on Japan for El Niño and an increase in landfall on South Korea for La Niña, although it is not a significant change in their study. Our results agree well with those of Ho et al. (2005), where the frequency of TC passages in different observed ENSO years is examined. Their study did not find the effect to be as significant, possibly because they examine only 7 years of each ENSO state based on a threshold of ENSO index.

Track density is now examined to help interpret these landfall-ENSO results. Density is defined as the number of 6-h track points per unit area, calculated by counting the track points in every $1^{\circ}$ grid box and dividing by the area of the grid box. The track densities for the two ENSO states for each version of the model are shown in Fig. 8. The results show large similarities between the simulations from the Genesis-Only (Figs. 8a,d) and Both (Figs. 8c,f) model versions: the southeastern peak in density during El Niño moves to the northwest during La Niña. This shift in track density is consistent with the increased La Niña landfall rates on Vietnam, China, the Korean Peninsula, and Japan seen in Fig. 7c. The density shift is a consequence of shifting genesis regions, and is not observed in the Track-Only model (Figs. 8b,e). However, the Track-Only model shows higher track density overall in either ENSO state, with a notable northward increase in track density, especially during El Niño states. 


\section{GENESIS-ONLY}

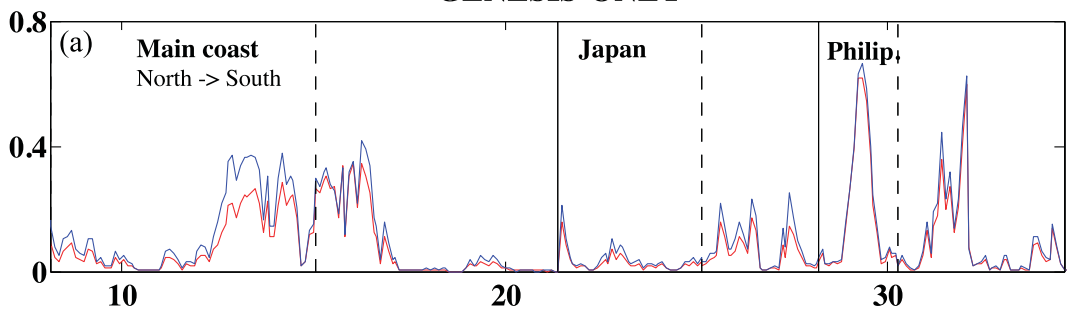

TRACK-ONLY
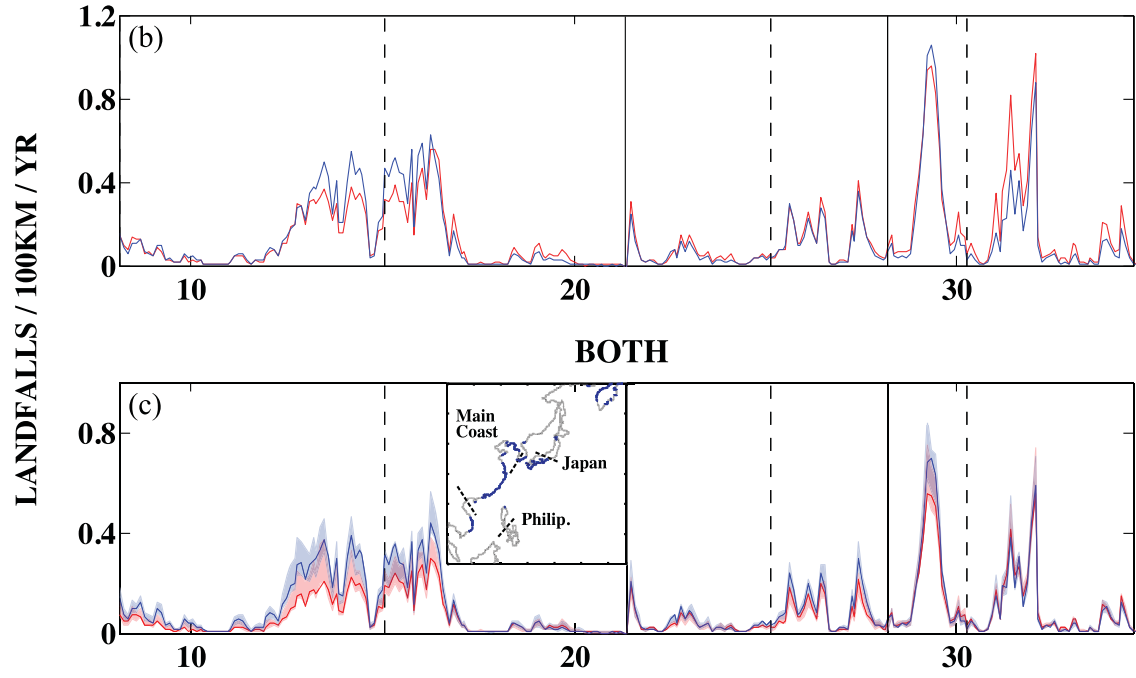

EN - LN LANDFALL RATES

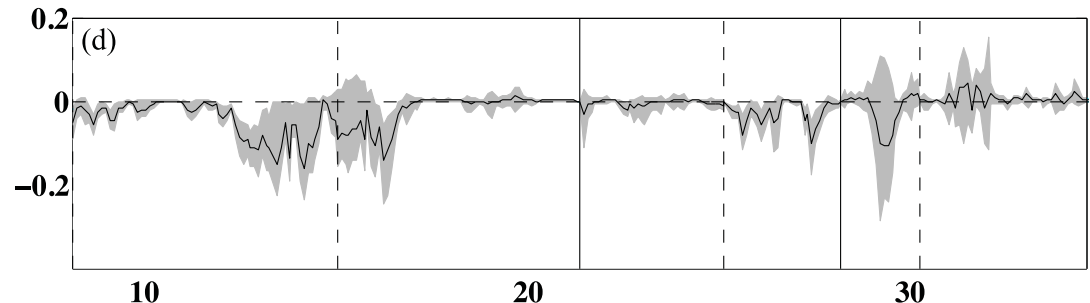

DISTANCE ALONG COAST IN 1000KM

FIG. 7. Ensemble-mean landfall rates for 1 -yr model runs for ENSO indices of +2 (red) and -2 (blue) are shown for the coasts of Japan, the Philippines, and the two southernmost regions of the main coast. Each panel represents a different model configuration: (a) Genesis-Only (only ENSO dependence in the genesis component), (b) Track-Only (only ENSO dependence in the track-propagation component), and (c) Both (ENSO dependence in both). (d) The landfall rate difference between the two ENSO states from the Both model. The shading in (c) and (d) indicates the jackknife uncertainty at the $95 \%$ confidence level. The coastal segments with significant increases in La Niña year landfall are indicated in blue in (c).

\section{Discussion}

We have constructed a statistical track model for TCs in the WNP with seasonal climate and ENSO dependence in the genesis and track-propagation components with the goal of estimating the ENSO influence on East Asian landfall rates. The model implements local regression using seasonally varying climate variables and the JASO Niño-3.4 index as predictors. The advantage of a basinwide track model such as this compared with analysis of landfalls directly is that it brings to bear orders of magnitude more data to landfall estimation, essentially projecting information from the entire basin onto coastal regions of interest for climate states of interest.

Stochastic simulations of TCs in extreme ENSO states reveal that ENSO affects landfall locations and frequency. We compute mean landfall rates and uncertainties about the means for strong El Niño and La Niña 
GENESIS-ONLY
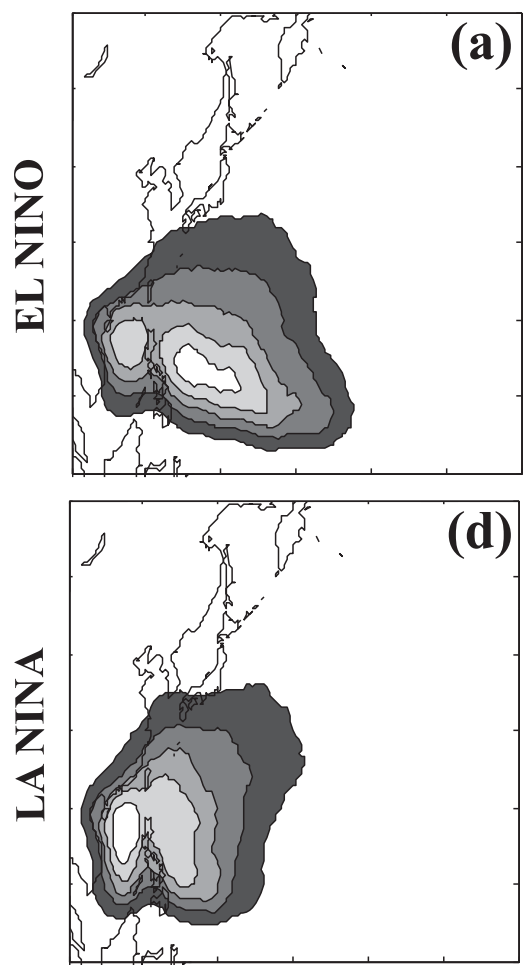

TRACK-ONLY
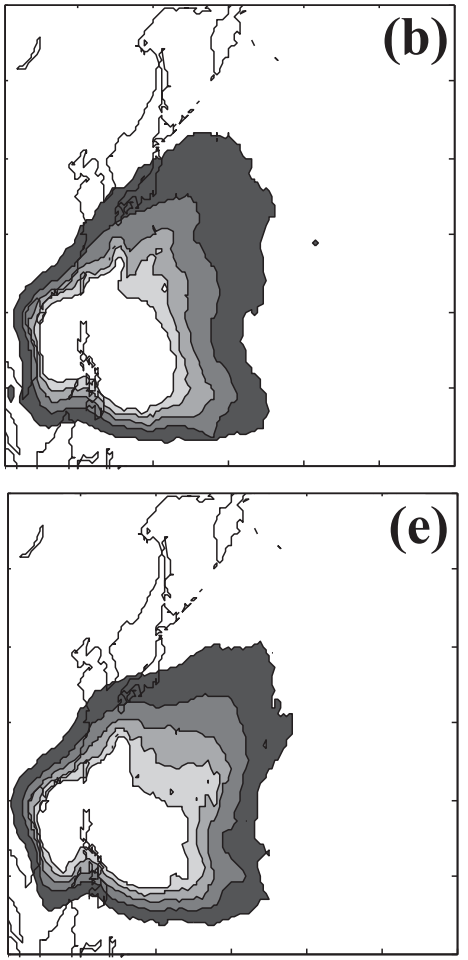

BOTH

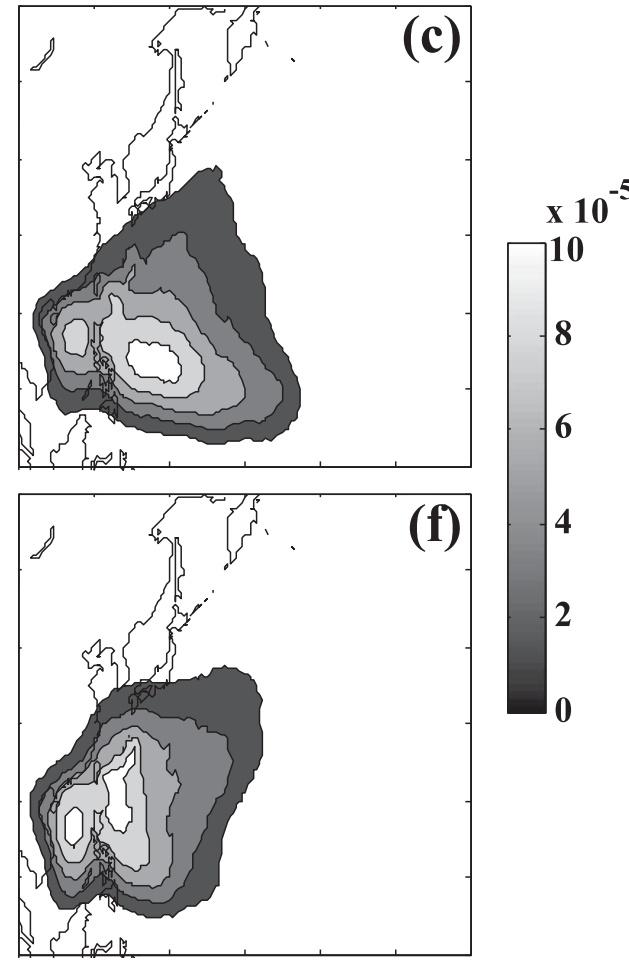

FIG. 8. Track densities in units of 6-hourly track points per square kilometer for the three different versions of the model for (a)-(c) El Niño and (d)-(f) La Niña years. Note the effects of the genesis region shift for the (left) Genesis-Only and (right) Both versions and the high track density in the South China Sea for the (center) Track-Only version that agrees with the higher landfall rates in simulations.

states. There are regions of significantly higher landfall rates in the Korean Peninsula, China, Vietnam, Japan, and the northern Philippines during La Niña. The majority of the ENSO-landfall relationship is mediated by the influence of ENSO on genesis rates and geographic distribution. However, ENSO influence on TC tracks adds and subtracts from the genesis-landfall effect in various coastal regions.

The model is also evaluated by comparing simulated landfall rates to direct historical landfalls. Overall, the model performs well: in most regions historical landfall rates are inside the spread of model results across large ensemble simulations, indicating a lack of bias. There are a few regions where the model has biased landfall rates. These biases have been reduced, however, compared with the YH11 model version, which had no seasonality and no ENSO-track effects. The performance in historical simulations also indicates that the model has skill in simulating TC landfalls for all ENSO states, including neutral and weak events. The fact that our model has skill while using relatively few predictors highlights the importance of ENSO and seasonal effects on WNP TCs.
Future directions for this work include developing a model component for the intensity and size of each TC, similar to Vickery and Twisdale (1995) and Vickery et al. (2000), to make a more complete risk analysis. Studies such as Irish et al. (2008) and Weisberg and Zheng (2006) show that it is the complex relationship between factors such as translational speed, storm size, intensity, bathymetry, and orientation that determines damage. Further, modeling of other TC-climate relationships may also be explored in the future where the model is dependent on other climate states. Advances in the understanding of the relationships between WNP TC activity and the Madden-Julian oscillation, quasi-biennial oscillation (Ho et al. 2009), central Pacific SST warming events (Kim et al. 2011), and even the more remote Antarctic Oscillation (Ho et al. 2005) and Arctic Oscillation (Choi and Byun 2010) motivate their additional consideration as statistical predictors to further improve the accuracy of our model.

Acknowledgments. This work was supported in part by a grant from the NASA Applied Sciences program. We thank Phil Klotzbach and two anonymous reviewers for their helpful comments on this work. 


\section{REFERENCES}

Ashok, K., S. K. Behera, S. A. Rao, H. Weng, and T. Yamagata, 2007: El Niño Modoki and its possible teleconnection. J. Geophys. Res., 112, C11007, doi:10.1029/2006JC003798.

Barnston, A. G., M. Chelliah, and S. B. Goldenberg, 1997: Documentation of a highly ENSO-related SST region in the equatorial Pacific. Atmos.-Ocean, 35, 367-383.

Camargo, S. J., and A. H. Sobel, 2005: Western North Pacific tropical cyclone intensity and ENSO. J. Climate, 18, 2996-3006.

- , K. A. Emanuel, and A. H. Sobel, 2007a: Use of a genesis potential index to diagnose ENSO effects on tropical cyclone genesis. J. Climate, 20, 4819-4834.

- A. W. Robertson, S. J. Gaffney, P. Smyth, and M. Ghil, 2007b: Cluster analysis of typhoon tracks. Part I: General properties. J. Climate, 20, 3635-3653.

,,,,---- and,$- 2007 \mathrm{c}$ : Cluster analysis of typhoon tracks. Part II: Large-scale circulation and ENSO. J. Climate, 20, 3654-3676.

— A. H. Sobel, A. G. Barnston, and P. J. Klotzbach, 2010: The influence of natural climate variability, and seasonal forecasts of tropical cyclone activity. Global Perspectives on Tropical Cyclones: From Science to Mitigation, 2nd ed. J. C. L. Chan and J. D. Kepert, Eds., World Scientific, 325-360.

Cane, M. A., 1991: Forecasting El Niño with a geophysical model. Teleconnections Linking Worldwide Climate Anomalies, M. H. Glantz, R. W. Katz, and N. Nicholls, Eds., Cambridge University Press, 345-370.

Chan, J. C. L., 1985: Tropical cyclone activity in the northwest Pacific in relation to the El Niño/Southern Oscillation phenomenon. Mon. Wea. Rev., 113, 599-606.

— 2000: Tropical cyclone activity over the western North Pacific associated with El Niño and La Niña Events. J. Climate, 13, 2960-2972.

— and W. M. Gray, 1982: Tropical cyclone movement and surrounding flow relationships. Mon. Wea. Rev., 110, 1354-1374.

— cific typhoon activity from an observational perspective. J. Climate, 17, 4590-4602.

Chia, H.-H., and C. F. Ropelewski, 2002: The interannual variability in the genesis location of tropical cyclones and the northwest Pacific. J. Climate, 15, 2934-2944.

Choi, K. S., and H. R. Byun, 2010: Possible relationship between western North Pacific tropical cyclone activity and Arctic Oscillation. Theor. Appl. Climatol., 100 (3-4), 261-274.

Clark, J. D., and P.-S. Chu, 2002: Interannual variation of tropical cyclone activity over the central North Pacific. J. Meteor. Soc. Japan, 80, 403-418.

Darling, R., 1991: Estimating probabilities of hurricane wind speeds using a large-scale empirical model. J. Climate, 4, 1035-1046.

Dong, K., 1988: El Niño and tropical cyclone frequency in the Australian region and northwest Pacific. Aust. Meteor. Mag., 36, 219-255.

Drayton, M., 2000: A stochastic, "basin-wide" model of Atlantic hurricanes. Proc. 24th Conf. on Hurricanes and Tropical Meteorology, Ft. Lauderdale, FL, Amer. Meteor. Soc., 17A.3. [Available online at https://ams.confex.com/ams/last2000/ webprogram/Paper12797.html.]

Elsner, J. B., and K. B. Liu, 2003: Examining the ENSO-typhoon hypothesis. Climate Res., 25, 43-54.

Emanuel, K., S. Ravela, E. Vivant, and C. Risi, 2006: A statistical deterministic approach of hurricane risk assessment. Bull. Amer. Meteor. Soc., 87, 299-314.
Fedorov, A. V., S. L. Harper, S. G. Philander, B. Winter, and A. Wittenberg, 2003: How predictable is El Niño? Bull. Amer. Meteor. Soc., 84, 911-919.

Goddard, L., and Coauthors, 2010: Providing seasonal-to-interannual climate information to risk management and decision-making. Procedia Environ. Sci., 1, 81-101.

Graf, M., K. Nishijima, and M. H. Faber, 2009: A probabilistic typhoon model for the northwest Pacific region. Proc. Seventh Asia-Pacific Conf. on Wind Engineering, Taipei, Taiwan, International Association for Wind Engineering, M2-B. [Available online at http://www.iawe.org/Proceedings/ 7APCWE/M2B_1.pdf.]

Hall, T. M., and S. Jewson, 2007: Statistical modeling of North Atlantic tropical cyclone tracks. Tellus, 59A, 486-498.

Ho, C.-H., J.-H. Kim, H.-S. Kim, C.-H. Sui, and D.-Y. Gong, 2005: Possible influence of the Antarctic Oscillation on tropical cyclone activity in the western North Pacific. J. Geophys. Res., 110, D19104, doi:10.1029/2005JD005766.

— , H.-S. Kim, J. H. Jeong, and S. W. Son, 2009: Influence of stratospheric quasi-biennial oscillation on tropical cyclone tracks in the western North Pacific. Geophys. Res. Lett., 36, L06702, doi:10.1029/2009GL037163.

Irish, J. L., D. T. Resio, and J. J. Ratcliff, 2008: The influence of storm size on hurricane surge. J. Phys. Oceanogr., 38, 20032013.

James, M. K., and L. B. Mason, 2005: Synthetic tropical cyclone database. J. Waterw. Port Ocean Eng., 131 (4), 181-192.

Kalnay, E., and Coauthors, 1996: The NCEP/NCAR 40-Year Reanalysis Project. Bull. Amer. Meteor. Soc., 77, 437-471.

Kim, H.-M., P. J. Webster, and J. A. Curry, 2011: Modulation of North Pacific tropical cyclone activity by three phases of ENSO. J. Climate, 24, 1839-1849.

Knapp, K. R., M. C. Kruk, D. H. Levinson, H. J. Diamond, and C. J. Neumann, 2010: The International Best Track Archive for Climate Stewardship (IBTrACS): Unifying tropical cyclone best track data. Bull. Amer. Meteor. Soc., 91, 363376.

Lander, M., 1994: An exploratory analysis of the relationship between tropical storm formation in the western North Pacific and ENSO. Mon. Wea. Rev., 122, 636-651.

Rumpf, J., H. Weindl, P. Höppe, P. E. Rauch, and V. Schmidt, 2007: Stochastic modeling of tropical cyclone tracks. Math. Methods Oper. Res., 66, 475-490.

Sabbatelli, T. A., and M. E. Mann, 2007: The influence of climate state variables on Atlantic tropical cyclone occurrence rates. J. Geophys. Res., 112, D17114, doi:10.1029/2007JD008385.

Saunders, M. A., R. E. Chandler, C. J. Merchant, and F. P. Roberts, 2000: Atlantic hurricanes and NW Pacific typhoons: ENSO spatial impacts on occurrence and landfall. Geophys. Res. Lett., 27, 1147-1150.

Smith, T. M., and R. W. Reynolds, 2004: Improved extended reconstruction of SST (1854-1997). J. Climate, 17, 2466-2477.

Tukey, J., 1958: Bias and confidence in not quite large samples. Ann. Math. Stat., 29, 614.

Vickery, P. J., and L. A. Twisdale, 1995: Wind field and filling models for hurricane wind-speed predictions. J. Struct. Eng., 121, 1700-1709.

, P. F. Skerlj, and L. A. Twisdale, 2000: Simulation of hurricane risk in the U.S. using empirical track model. J. Struct. Eng., 126, 1222-1237.

Vitart, F., 2006: Seasonal forecasting of tropical storm frequency using a multi-model ensemble. Quart. J. Roy. Meteor. Soc., 132, 647-666. 
—, and Coauthors, 2007: Dynamically-based seasonal forecasts of Atlantic tropical storm activity issued in June by EUROSIP. Geophys. Res. Lett., 34, L16815, doi:10.1029/ 2007GL030740.

Wang, B., and J. C. L. Chan, 2002: How strong ENSO events affect tropical storm activity over the western North Pacific. J. Climate, 15, 1643-1658.

— , R. Wu, and X. Fu, 2000: Pacific-East Asian teleconnection: How does ENSO affect East Asian climate? J. Climate, 13, $1517-1536$

Weisberg, R. H., and L. Zheng, 2006: Hurricane storm surge simulations for Tampa Bay. Estuaries Coasts, 29, 899-913.

Wu, M. C., W. L. Chang, and W. M. Leung, 2004: Impacts of El Niño-Southern Oscillation events on tropical cyclone landfalling activity in the western North Pacific. J. Climate, 17, 1419-1428.

Yin, J., M. B. Welch, H. Yashiro, and M. Shinohara, 2009: Basinwide typhoon risk modeling and simulation for western North Pacific basin. Proc. Seventh Asia-Pacific Conf. on Wind Engineering, Taipei, Taiwan, International Association for Wind Engineering, M2-B. [Available online at http://www.iawe.org/ Proceedings/7APCWE/M2B_2.pdf.]

Yonekura, E., and T. M. Hall, 2011: A statistical model of tropical cyclone tracks in the western North Pacific with ENSOdependent cyclogenesis. J. Appl. Meteor. Climatol., 50,1725-1739.

Zhang, W., H.-F. Graf, Y. Leung, and M. Herzog, 2012: Different El Niño types and tropical cyclone landfall in East Asia. J. Climate, 25, 6510-6523. 